Check for updates

Cite this: Phys. Chem. Chem. Phys., 2020, 22, 8781
Received 25th July 2019,

Accepted 21st March 2020

DOI: $10.1039 / c 9 c p 04158 c$

rsc.li/pccp

\section{Lipid vesicle composition influences the incorporation and fluorescence properties of the lipophilic sulphonated carbocyanine dye SP-DiO†}

\author{
Quentin Lubart, ${ }^{a b}$ Jonas K. Hannestad, (D) ${ }^{a}$ Hudson Pace, (D) ${ }^{c}$ Daniel Fjällborg, ${ }^{a}$ \\ Fredrik Westerlund, (DD ${ }^{\mathrm{b}}$ Elin K. Esbjörner ${ }^{\mathrm{b}}$ and Marta Bally (D) *d
}

\begin{abstract}
Lipophilic carbocyanine dyes are widely used as fluorescent cell membrane probes in studies ranging from biophysics to cell biology. While they are extremely useful for qualitative observation of lipid structures, a major problem impairing quantitative studies is that the chemical environment of the lipid bilayer affects both the dye's insertion efficiency and photophysical properties. We present a systematic investigation of the sulphonated carbocyanine dye 3,3'-dioctadecyl-5,5'-di(4-sulfophenyl) (SP-DiO) and demonstrate how its insertion efficiency into pre-formed lipid bilayers and its photophysical properties therein determine its apparent fluorescence intensity in different lipid environments. For this purpose, we use large unilamellar vesicles (LUVs) made of lipids with distinct chain unsaturation, acyl chain length, head group charge, and with variation in membrane cholesterol content as models. Using a combination of absorbance, fluorescence emission, and fluorescence lifetime measurements we reveal that SP-DiO incorporates more efficiently into liquid disordered phases compared to gel phases. Moreover, incorporation into the latter phase is most efficient when the mismatch between the length of the lipid and dye hydrocarbon chains is small. Furthermore, SP-DiO incorporation is less efficient in LUVs composed of negatively charged lipids. Lastly, when cholesterol was included in the LUV membranes, we observed significant spectral shifts, consistent with dye aggregation. Taken together, our study highlights the complex interplay between membrane composition and labeling efficiency with lipophilic dyes and advocates for careful assessment of fluorescence data when attempting a quantitative analysis of fluorescence data with such molecules.
\end{abstract}

\section{Introduction}

Fluorescent lipophilic dyes are useful for non-covalent labeling of lipid membrane structures through their partitioning into the lipid phase where they establish strong hydrophobic interactions with the hydrocarbon tails of phospholipids. Accordingly, various membrane dyes have become widely used in cell biology, particularly in fluorescence microscopy applications. ${ }^{1}$ Additionally, these dyes have proven invaluable for in vitro studies aimed at understanding the biophysics of synthetic lipid membranes as well as of live cells, where membrane

\footnotetext{
${ }^{a}$ Division of Biological Physics, Department of Physics, Chalmers University of Technology, 41296 Gothenburg, Sweden

${ }^{b}$ Division of Chemical Biology, Department of Biology and Biological Engineering, Chalmers University of Technology, 41296 Gothenburg, Sweden

${ }^{c}$ Department of Integrative Medical Biology, Umeå University, 90185 Umeå, Sweden

${ }^{d}$ Department of Clinical Microbiology \& Wallenberg Centre for Molecular Medicine, Umeå University, 90185 Umeå, Sweden. E-mail: marta.bally@umu.se

$\dagger$ Electronic supplementary information (ESI) available. See DOI: 10.1039/ с9ср04158c
}

properties such as heterogeneity, lateral domain formation or fluidity have being investigated. ${ }^{2-5}$ Recently, there has also been an increased interest in using lipophilic dyes to efficiently and reproducibly label both synthetic and biological nanoscale lipid vesicles such as liposomes, ${ }^{6}$ cell-secreted extracellular vesicles $^{7}$ and viruses. ${ }^{6-14}$

The photophysical properties of lipophilic membrane dyes are often greatly affected by the chemical environment and the surrounding lipids. ${ }^{15-17}$ Moreover, the exact lipid composition of the membrane, including its charge and fluidity, can strongly influence the insertion efficiency of the dye and hence its effective concentration in a particular lipid vesicle. ${ }^{17-19}$ As a consequence, fluorescence intensities may vary greatly between samples with the same vesicle concentration and direct quantitative comparison of fluorescence data measured in different lipid systems becomes cumbersome. This in turn makes the use of extrinsic labelling to stain cell-derived vesicles, where the lipid composition and putative vesicle-to-vesicle heterogeneities are generally unknown, highly challenging and ambiguous. A more in-depth understanding of how the chemical composition of a 
(a)

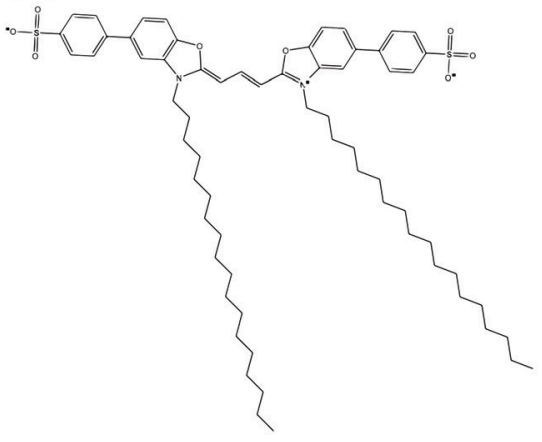

(b)

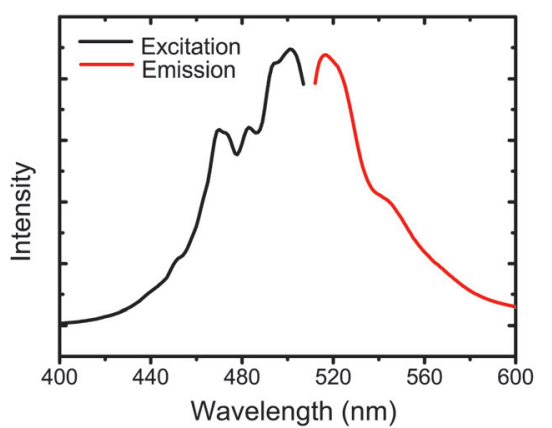

(c)

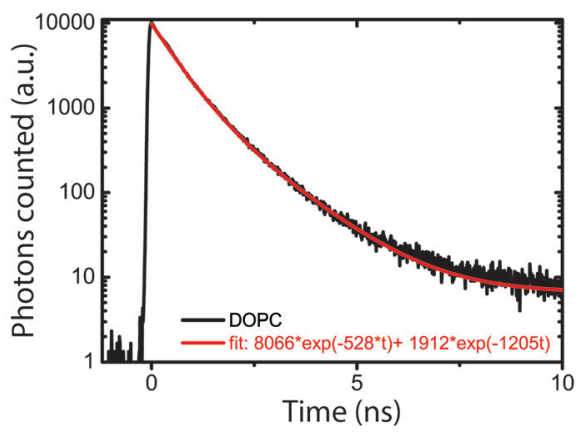

Fig. 1 Molecular structure and fluorescence properties of the lipophilic SP-DiO dye. (a) Molecular structure representation of 3,3'-dioctadecyl-5,5'di(4-sulfophenyl)oxacarbocyanine (SP-DiO). (b) Excitation (black) and emission (red) spectra of SP-DiO incorporated into 1,2-dioleoyl-sn-glycero-3phosphocholine (DOPC) LUVs. (c) Time-correlated single photon counting (TCSPC) fluorescence decay curve of SP-DIO in DOPC LUVs.

lipid membrane affects labeling efficiency and the fluorescent properties of lipophilic dyes is therefore needed. Furthermore, systematic knowledge of the interplay between dye properties and lipid composition lead to new strategies for selective labeling of specific cellular membrane compartments.

In this study, we have systematically investigated how lipid unsaturation, phospholipid acyl chain length, head-group charge and cholesterol content of the membrane influence the insertion and fluorescent properties of the commercially available lipophilic and sulphonated carbocyanine dye SP-DiO C18(3) (3,3'-dioctadecyl-5,5'-di(4-sulfophenyl)). ${ }^{20}$ This dye, as well as other members of the carbocyanine dye family, have been widely used to label cell membranes ${ }^{21-23}$ viruses, ${ }^{9,10,24}$ exosomes ${ }^{12,25,26}$ and synthetic liposomes. ${ }^{27-31}$ From a structural point of view, lipophilic carbocyanine dyes, are symmetric molecules consisting of two aromatic cyanine moieties joined by three methine groups, which together constitute the fluorophore part of the molecule. Furthermore, to introduce lipophilicity, alkyl chains are conjugated to each cyanine moiety as depicted in Fig. 1a. The SP-DiO $\mathrm{C}_{18}(3)$ (hereafter referred to as SP-DiO) used here additionally carries two sulphonate groups to improve its water solubility compared to the DiO counterpart, ${ }^{32}$ thereby facilitating labeling in aqueous environments. However, this chemical group also confers the dye with a net negative charge, which could influence its interactions with lipid head groups, especially since the chromophore part is located close to the membrane surface ${ }^{33}$ or in the interface region. ${ }^{34}$

\section{Experimental}

\subsection{Materials}

All materials were purchased from commercial sources, unless stated otherwise. We obtained 1,2-dioleoyl-sn-glycero-3-phosphoL-serine (DOPS), 1,2-dioleoyl-sn-glycero-3-phosphocholine (DOPC), 1-palmitoyl-2-oleoyl-glycero-3-phosphocholine (POPC), 1,2-distearoylsn-glycero-3-phosphocholine (DSPC), 1,2-dipalmitoyl-sn-glycero3-phosphocholine (DPPC), 1,2-dimyristoyl-sn-glycero-3-phosphocholine (DMPC) and cholest-5-en-3 $\beta$-ol (Cholesterol) from Avanti Polar Lipids (Alabaster, AL). Phosphate-buffered saline
(PBS, tablets) and 3,3'-dioctadecyl-5,5'-di(4-sulfophenyl)oxacarbocyanine (SP-DiO $\mathrm{C}_{18}(3)$ ) were purchased from Sigma-Aldrich (Stockholm, Sweden). Illustra MicroSpin S-200 HR columns were from GE Healthcare (Stockholm, Sweden). PBS was filtered with Millipore filters $(0.1 \mu \mathrm{m})$ before use (Merck, Stockholm, Sweden). Water was deionized and filtered using a Milli-Q system (Merck, Stockholm, Sweden).

\subsection{Vesicle preparation}

Large unilamellar vesicles (LUVs) were prepared by drying the appropriate lipid mixture overnight in vacuum. The lipid films were then hydrated in PBS buffer at appropriate temperature $\left(70{ }^{\circ} \mathrm{C}\right.$ for DSPC and DPPC, $50{ }^{\circ} \mathrm{C}$ for DMPC and room temperature for all other formulations). Following this, lipid emulsions were subjected to extrusion using a mini-extruder (Avanti Polar Lipids), with two polycarbonate filters of $100 \mathrm{~nm}$ pore diameter (21 passages). A second extrusion through filters of $30 \mathrm{~nm}$ pore diameter (5 passages) was applied on all the formulations, except for DPPC and DSPC.

\subsection{Large unilamellar vesicle (LUV) labelling}

The pre-formed LUVs were labelled with SP-DiO prior to each experiment. $2 \mu \mathrm{L}$ of SP-DiO dye (from a $400 \mu \mathrm{M}$ stock solution in DMSO) was mixed with $18 \mu \mathrm{L}$ of LUVs (nominal concentration of $2 \mathrm{mg} \mathrm{mL}^{-1}$ ) to achieve a final dye concentration of $40 \mu \mathrm{M}$. The samples were incubated for $50 \mathrm{~min}$, protected from light, to allow the dye to partition into the lipid bilayers of the LUVs. The mixtures were then centrifuged for $2 \mathrm{~min}$ at $700 \mathrm{rpm}$ through illustra MicroSpin S200 HR columns to remove excess unbound dye. The columns were pre-equilibrated in PBS buffer.

\subsection{Absorption spectroscopy}

Absorption spectra were recorded on a Cary 5000 UV-Vis spectrometer (Agilent Technologies) with a scan rate of $600 \mathrm{~nm} / \mathrm{min} .10 \mu \mathrm{L}$ of labelled LUVs obtained as described above, were mixed with $90 \mu \mathrm{L}$ PBS buffer in a quartz cuvette with $3 \mathrm{~mm}$ path length. All spectra were corrected for background contributions by subtracting appropriate blanks. 


\subsection{Fluorescence spectroscopy}

Fluorescence spectra were recorded on a QM-4/2005 spectrofluorometer (Photon Technology International Inc., Birmingham, NJ). $10 \mu \mathrm{L}$ of labelled vesicles were mixed with $90 \mu \mathrm{L}$ PBS buffer in a quartz cuvette $[0.7 \mathrm{~mL}, 10 \mathrm{~mm}]$. Three spectra were recorded and averaged. SP-DiO was first excited at the absorption maximum $494 \mathrm{~nm}$ to determine the exact positions of the emission peaks. Then, excitation spectra were recorded with the emission monochromator set to the emission maximum for each lipid vesicle type (516 nm for DMPC, $519 \mathrm{~nm}$ for DPPC, $542 \mathrm{~nm}$ for POPC/Chol 7/3 and $517 \mathrm{~nm}$ for all other compositions; see ESI $\dagger$ Table S1). Finally, the emission peaks were recorded by exciting each sample at its respective excitation maximum, which was $501 \mathrm{~nm}$ for DOPC, DOPS, POPC and POPC/Chol 9/1, $503 \mathrm{~nm}$ for DSPC and DMPC, $504 \mathrm{~nm}$ for POPC/Chol 7/3 and $507 \mathrm{~nm}$ for DPPC. The excitation and emission bandpass were both set to $1 \mathrm{~nm}$.

\subsection{Time-correlated single photon counting (TCSPC)}

Time-resolved fluorescence decays were recorded to determine the SP-DiO fluorescence lifetimes in the different LUVs, using time correlated single photon counting (TCSPC). An approximately 90 ps excitation pulse was provided using a pulsed laser diode with a $483 \mathrm{~nm}$ center wavelength (LDH-PC485B, PicoQuant). The excitation light was passed through a filter (482/ $18 \mathrm{~nm}$ BrightLine ${ }^{\mathbb{R}}$ single-band bandpass filter, Semrock) to remove any red-shifted components from before illuminating the sample. Fluorescence emission from the sample was collected at $517 \mathrm{~nm}(\Delta \lambda=10 \mathrm{~nm})$ and detected using a thermoelectrically cooled microchannel plate photomultiplier tube detector (R3809U-50, Hamamatsu). The signal was digitalized using a multichannel analyzer with 4096 channels (SPC-300, Edinburgh Analytical Instruments).

To provide average lifetime values for comparison of lifetimes across the samples, the fluorescence decays for all investigated samples were fitted with double exponential expressions between 0 and $10 \mathrm{~ns}$. The quality of the fit was evaluated by minimizing $\chi^{2}$ values.

\subsection{Nanoparticle tracking analysis measurements}

Nanoparticle tracking analysis data (NTA) were obtained using a NanoSight LM10 equipped with a $488 \mathrm{~nm}$ laser. All measurements were performed in scattering mode, under a flow rate: $100(\sim 10 \mu \mathrm{L} / \mathrm{min})$ using a Nanosight syringe pump module; the buffer viscosity was considered to be that of water. Each sample was recorded with a collection of three movies of 90 seconds each. The movies were analyzed with the software NTA 3.2 (Malvern Instruments Ltd, Malvern, UK) with camera level: 15 and detection threshold: 3 to be able to determine optimized size distributions and concentrations. Comparative measurements were done on the same day and using the same protocol.

The total surface area (TSA) was calculated with the following equation:

$$
\sum_{n=1 \ldots}^{1000} 4 \pi r_{n}^{2} c_{n}
$$

where $r_{n}$ is the particle radius and $c_{n}$ the concentration of particles with radius $r_{n}$.

\subsection{Labeling of native membrane fractions with SP-DiO}

Native membrane vesicles were produced by first growing HeLa cells to confluency in DMEM media. The cells were washed and scraped using cold HEPES buffer saline (HBS, $25 \mathrm{mM}$ HEPES $150 \mathrm{mM} \mathrm{NaCl}$, Complete protease inhibitor cocktail (Roche)), then pelleted. The cell pellet was resuspended in HBS and cells were ruptured using 3 passes through a cell disruptor (Constant Systems). Unruptured cells and nuclei were removed by differential centrifugation, $3200 \times g$ for $10 \mathrm{~min}$ at $4{ }^{\circ} \mathrm{C}$. Mitochondrial membranes were then removed by differential centrifugation, $6000 \times g$ for $20 \mathrm{~min}$ at $4{ }^{\circ} \mathrm{C}$, using a Beckman Ti70 rotor. The supernatant was collected and the membrane fraction was pelleted using a Beckman Ti70 rotor, $180000 \times \mathrm{g}$ for $90 \mathrm{~min}$ at $4{ }^{\circ} \mathrm{C}$. This fraction was either treated with Triton X-100 for $1 \mathrm{~h}$ at $4{ }^{\circ} \mathrm{C}$ or untreated. Then, both treated and untreated membrane fraction solutions were mixed with sucrose to $40 \%$ and layered at the bottom of a discontinuous gradient with sections of 30 and $5 \%$ sucrose. The gradients were centrifuged using a Beckman SW41 rotor $\left(251000 \times g\right.$ for $2.5 \mathrm{~h}$ at $\left.4{ }^{\circ} \mathrm{C}\right)$. The material focused into an opaque band at the interface of the 5 and $30 \%$ sucrose regions was collected. In order to label both native vesicle types with SP-DiO, $4 \mu \mathrm{L}$ of SP-DiO dye (from a $400 \mu \mathrm{M}$ stock solution in DMSO) was mixed with $36 \mu \mathrm{L}$ of membrane material to achieve a final dye concentration of $40 \mu \mathrm{M}$. The samples were incubated for $50 \mathrm{~min}$ on ice, protected from light. The mixtures were then centrifuged for $2 \mathrm{~min}$ at $700 \mathrm{rpm}$ through illustra MicroSpin S200 HR columns to remove excess unbound dye. The columns were preequilibrated in HBS buffer.

\section{Results and discussion}

To gain insights into the influence of lipid composition on the membrane binding and fluorescence of the lipophilic carbocyanine dye SP-DiO, we prepared LUVs from a range of synthetic lipids (Fig. 2) and labelled them extrinsically, i.e. after vesicle preparation, in order to mimic labelling of biological vesicles (e.g., exosomes or viruses). A parallel study reveals that dye incorporation into LUVs of simple composition does not depend on vesicle size. ${ }^{35}$ Nevertheless, to make sure that our findings are not influenced by vesicle size, we ensured by NTA that all vesicles used in this study had highly similar average diameters and size distributions (ESI, $\dagger$ Fig. S4). The different vesicles were labeled by mixing SP-DiO and pre-made LUVs. The free dye remaining in solution was efficiently removed using spin-column size exclusion chromatography (ESI, $\dagger$ Fig. S5). The vesicle labeling step was carried out using similar dye-to-lipid ratios for all samples. The ratios were kept low enough to yield incorporated dye-to-lipid molar ratios well below 1\% (ESI, $\dagger$ Table S3) so that all experiments could be performed at conditions where dye-dye interaction in the membrane are expected to be low or non-existing. ${ }^{16}$ Absorption spectroscopy was used to determine the amount of bound dye, which was found to vary significantly depending on 


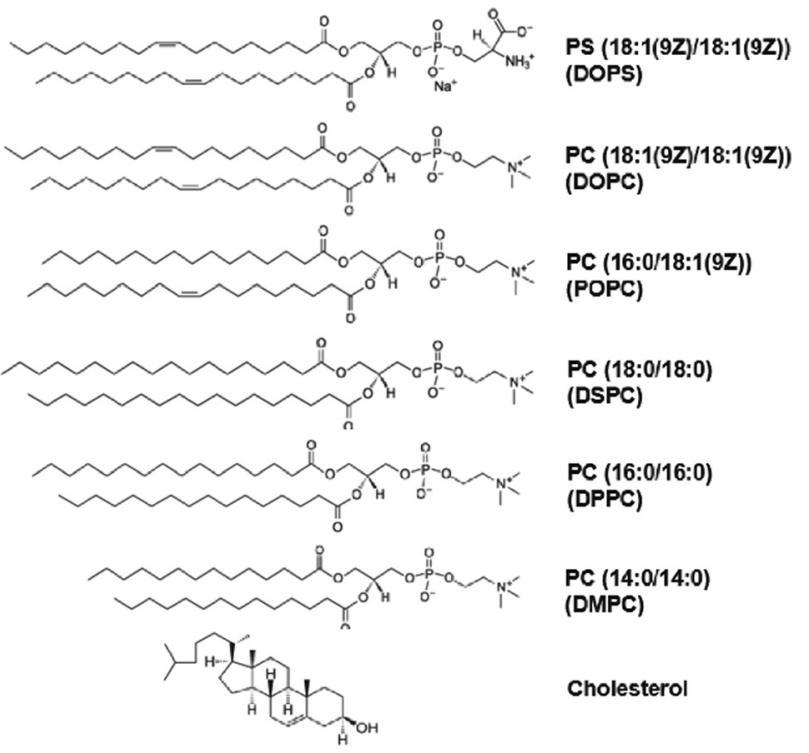

Fig. 2 Lipid structure. Molecular structure representation of the different lipids used in this study. From the top: 1,2-dioleoyl-sn-glycero-3-phosphoL-serine (DOPS), 1,2-dioleoyl-sn-glycero-3-phosphocholine (DOPC), 1-palmitoyl-2-oleoyl-glycero-3-phosphocholine (POPC), 1,2-distearoylsn-glycero-3-phosphocholine (DSPC), 1,2-dipalmitoyl-sn-glycero-3phosphocholine (DPPC), 1,2-dimyristoyl-sn-glycero-3-phosphocholine (DMPC) and cholest-5-en-3 $\beta$-ol (cholesterol).

lipid composition. We showed, in separate control experiments using LUVs where the SP-DiO dye was incorporated at the stage of lipid film preparation, that the molar extinction coefficient of SP-DiO was independent of the lipid environment (ESI, $\dagger$ Fig. S6), a finding also in good agreement with the observation that the absorption spectra profile of SP-DiO are similar for all monocomponent LUVs (ESI, $\uparrow$ Fig. S1). This further verifies that absorption is directly proportional to the concentration of bound dye. The different labelled LUV samples were thereafter subjected to steady state and time-resolved fluorescence analysis to record the excitation and emission profiles of the dye in LUVs with different compositions, as well as to quantify the ensemble-averaged emission intensities and the mean fluorescence lifetimes. Fluorescence analysis together with absorption data provides unique insights into the binding and brightness of the SP-DiO dye bound to LUVs. In particular, we use a relative quantum yield defined as $Q_{\mathrm{rel}}=I / c$, where $I$ is the integrated intensity of the fluorescence emission spectrum and $c$ the dye concentration obtained from $c=(A / l \times \varepsilon)$ where $A$ is the absorbance at $\lambda_{\mathrm{ex}}, 1$ the optical path length and $\varepsilon$ the molar extinction coefficient, and compare it to the fluorescence life time $\left(\tau_{\mathrm{av}}\right)$ to gain information about the photophysical behavior of the various systems. Both $Q_{\mathrm{rel}}$ and $\tau_{\mathrm{av}}$ are measures of the brightness of individual fluorophores. However, $\tau_{\mathrm{av}}$ only represent those fluorophores that emit light upon excitation while the value of $Q_{\text {rel }}$, which is essentially the ratio between photons emitted and absorbed, is also influenced also the presence of a non-emitting dye population. Therefore, in situations where changes in fluorescence intensity of a dye upon incorporation into different lipid environments are due to dynamic (excited state) quenching, $\tau_{\text {av }}$ is expected to change in relation to $Q_{\mathrm{rel}}$. If $\tau_{\mathrm{av}}$ and $Q_{\mathrm{rel}}$ are not changing proportionally, changes in intensity may be due to static (ground-state) quenching, suggesting presence of a non-fluorescing population of the dye. Our results are first discussed in general terms (Section 3.1) and then with respect to specific lipid properties (Sections 3.2-3.5)

\subsection{General properties of membrane-bound SP-DiO}

We first determined the excitation $\left(\lambda_{\mathrm{ex}}\right)$ and emission $\left(\lambda_{\mathrm{em}}\right)$ maxima of the SP-DiO dye as function of different lipid compositions. For all compositions, with exception of the cholesterol-containing LUVs, we found that the excitation and emission maxima were not significantly affected by the lipid chemistry in the LUVs. The excitation maximum was typically $\sim 501 \mathrm{~nm}$ and the emission maximum $\sim 517 \mathrm{~nm}$ as exemplified in Fig. 1b (see ESI, $\dagger$ Table S1 for all $\lambda_{\text {exc }}$ and $\lambda_{\text {em }}$ values). Increased amounts of cholesterol (46 mol\%) did, however, have a profound effect on the photophysical properties of SP-DiO; for this LUV type, $\lambda_{\mathrm{em}}$ was red-shifted by $>20 \mathrm{~nm}$ (see Section 3.5). Generally, SP-DiO appeared to be more efficient at providing strong fluorescent signals than the non-sulphonated counterpart DiO C18(3), further highlighting the benefits of adding the two sulfphophenyl groups when labeling membrane structures (ESI, $\dagger$ Fig. S7).

Next, we recorded time-resolved fluorescence decays for SP-DiO incorporated into the different LUVs using timecorrelated single photon counting. In all cases, the decays were multi-exponential, as exemplified in Fig. 1c; the complete set of decay curves are shown in ESI, $\dagger$ Fig. S3. Multiexponential decays have previously been reported for lipophilic cyanine dyes $^{16}$ and are likely associated with energy-level heterogeneities or excited state-reactions, rather than any heterogeneity inferred by the lipids. We found that the recorded decays were adequately represented by fitting with a double exponential decay function resulting in a shorter lifetime of $\sim 500-800 \mathrm{ps}$ and a longer lifetime of $\sim 1500-2000 \mathrm{ps}$; the shorter component was dominant in all cases (ESI, $\dagger$ Table S2).

\subsection{Acyl chain unsaturation}

To investigate the influence of lipid acyl chain unsaturation on the binding (incorporation) and fluorescence of SP-DiO, we used LUVs composed of the following lipids with at least one acyl chain with 18 carbons: DSPC (no unsaturation), POPC (one unsaturation) or DOPC (two unsaturations, one on each acyl chain) (Fig. 2 and Table 1). The presence of cis-unsaturation in the acyl chain of lipids is well-known to hinder lipid packing in the membrane, ${ }^{36,37}$ leading to a decrease in phase-transition temperature. The fully saturated lipid, DSPC, has a phase transition temperature $\left(T_{\mathrm{m}}\right)$ of $55{ }^{\circ} \mathrm{C}$ and the membranes of LUVs prepared with $100 \%$ of this lipid are therefore in the gel phase at room temperature, a phase characterized by essentially immobile lipids $\left(\sim 10^{-4}\right.$ to $\left.10^{-5} \mu \mathrm{m}^{2} \mathrm{~s}^{-1}\right) .^{38}$ POPC and DOPC, on the other hand, are both in a liquid disordered $\left(\mathrm{L}_{\mathrm{d}}\right)$ phase at room temperature, where the lipid diffusion coefficient for both is comparable $\left(\sim 10 \mu \mathrm{m}^{2} \mathrm{~s}^{-1}\right)$, but slightly higher 
Table 1 Lipid properties. Overview of the physico-chemical properties of the lipids used in this study. $L_{\circ}$ : liquid ordered; $L_{d}$ : liquid disordered; Chol: Cholesterol

\begin{tabular}{llllll}
\hline Formulation & Phase transition temp. $\left(T_{\mathrm{m}}\right)$ & Acyl-chain length & Unsaturation & Net charge & Lipid phase at 21 ${ }^{\circ} \mathrm{C}$ \\
\hline DOPS & $-11{ }^{\circ} \mathrm{C}$ & 18 & 2 & -1 & 0 \\
DOPC & $-17{ }^{\circ} \mathrm{C}$ & 18 & 2 & 0 & $\mathrm{~L}_{\mathrm{d}}$ \\
POPC & $-2{ }^{\circ} \mathrm{C}$ & $18 / 16$ & 1 & 0 & $\mathrm{~L}_{\mathrm{d}}$ \\
DSPC & $55{ }^{\circ} \mathrm{C}$ & 18 & 0 & 0 & $\mathrm{G}$ \\
DPPC & $41{ }^{\circ} \mathrm{C}$ & 16 & 0 & 0 & $\mathrm{Gel}$ \\
DMPC & $24{ }^{\circ} \mathrm{C}$ & 14 & 0 & 0 & $\mathrm{Gel}$ \\
POPC/Chol (18\%) & - & - & 0 & 0 & $\mathrm{~L}_{\mathrm{d}}+\mathrm{L}_{\mathrm{o}}$ \\
POPC/Chol (46\%) & - & - & & $\mathrm{L}_{\mathrm{o}}$
\end{tabular}

for DOPC. ${ }^{37}$ Absorbance measurements, following spin column separation of remaining unbound dye, revealed that the presence of lipid unsaturation had a significant effect on SP-DiO incorporation into the lipid vesicle membranes. As shown in Fig. 3a, the absorbance of SP-DiO is highest with DOPC vesicles, followed by POPC (one unsaturation) and DSPC (no unsaturation). Comparison of the absorption values at the maximum wavelength in the absorption spectra suggests that under identical labelling conditions, the amount of dye incorporated into DOPC vesicles is $\sim 2.5$-times higher than for POPC and $\sim 5$-times higher than for DSPC (Fig. 3b). Next, we compared the fluorescence intensity of the LUVs as measured by fluorescence spectroscopy (Fig. 3c and d). There is a clear and expected trend in the data that the LUVs with highest SP-DiO absorption (Fig. 3a and b) and hence highest incorporation of the dye, also exhibit the highest SP-DiO emission intensity (Fig. 3c and d), but the relation is not linear. To further analyze the relative contributions of the labeling efficiency versus the influence of the lipid environment, we use the relative quantum yield $\left(Q_{\text {rel }}=I / c\right)$. Since all absorbance measurements are performed under the same experimental conditions, $Q_{\text {rel }}$ allows for a direct qualitative comparison of the relative SP-DiO dye-concentration independent of the fluorescence intensity of SP-DiO in the different LUVs. We found that $Q_{\text {rel }}$ for the three LUV types discussed here (Fig. 3e) are similar for DSPC and POPC, but $\sim 1.5$ times lower for DOPC. This difference in $Q_{\text {rel }}$ correlates (a)

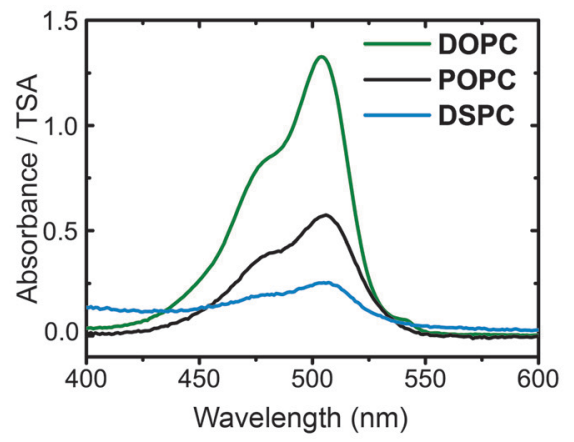

(b)

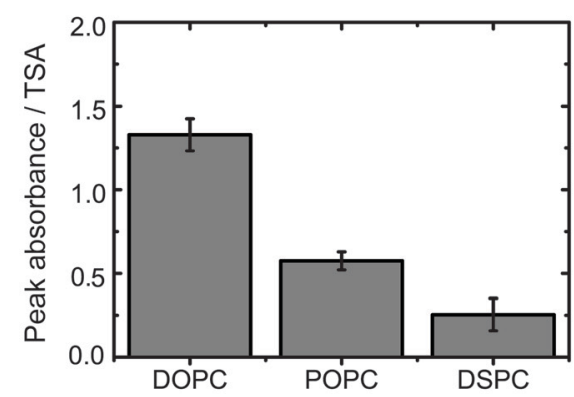

(c)



(d)

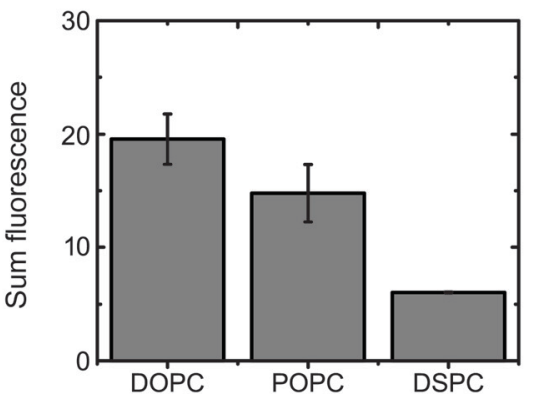

(e)

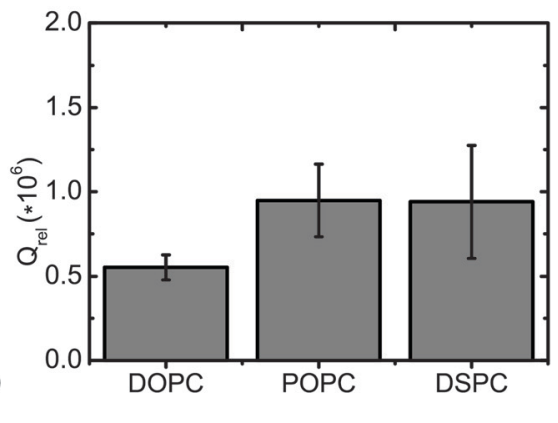

(f)

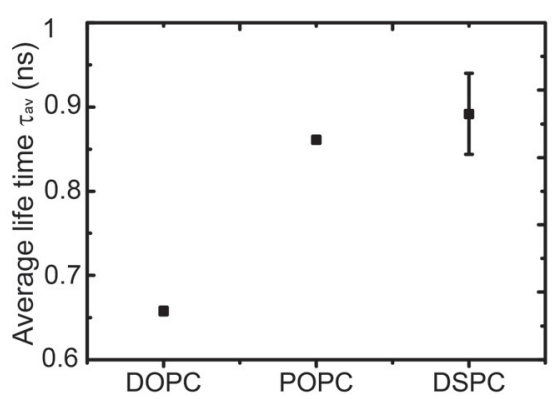

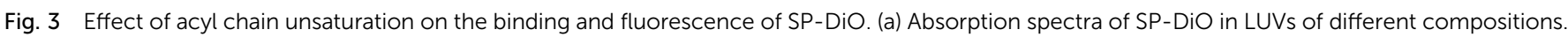

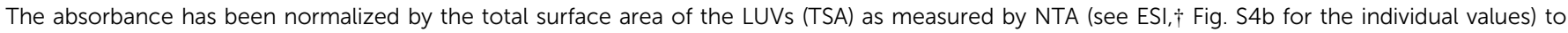

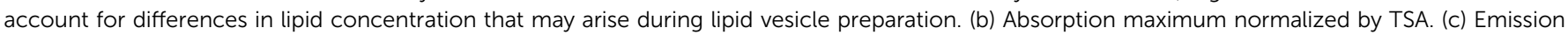

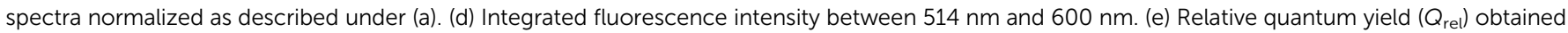

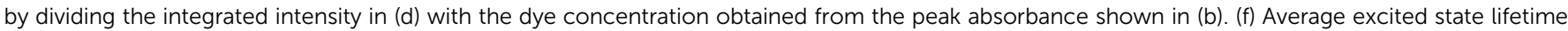

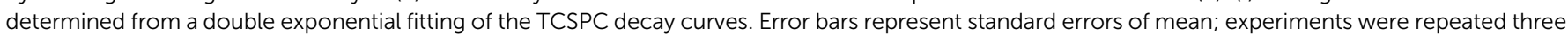
times $(n=3)$ on different LUV batches. 
qualitatively with the average fluorescence lifetimes $\left(\tau_{\mathrm{av}}\right)$ for DOPC, confirming that SP-DiO exhibits a distinctively different photophysical behavior in DOPC compared to the other two lipids, consistent with dynamic fluorescence quenching in the latter case. The highest $\tau_{\mathrm{av}}$ for the gel-phase membrane (DSPC) compared to the fluid ones is in agreement with previous reports on the structurally similar dye DiIC18(3) ${ }^{16}$ and could be ascribed to an increase in local viscosity preventing trans-cis photoisomerisation of the methine bridge, which would otherwise result in a non-radiative relaxation to the ground state. ${ }^{39}$ Alternatively, differences in orientation and penetration of the dye molecules into the bilayer could affect its spectral properties, as suggested for other environmentally sensitive dyes. ${ }^{40,41}$

Taken together, our results suggest that SP-DiO incorporates most efficiently into DOPC membranes, whereas the fluorescence lifetime is highest in DSPC membranes. Therefore, the higher labeling efficiency with DOPC is counter-balanced by a considerably weaker fluorescence of the SP-DiO dye in a DOPC membrane and vice versa, clearly demonstrating how both binding and brightness contributes to the total fluorescence intensity of the labelled membrane. Our result that SP-DiO incorporates more efficiently in DOPC compared to POPC, and DSPC LUVs suggests that the dye is more easily inserted in a lipid bilayer with high fluidity, a hypothesis further confirmed by control experiments showing an increase in dye incorporation for gel phase liposomes, if labeling is performed above the phase transition temperature (data not shown). At first glance, this appears to be in contrast with reports on the structurally related dye DiIC18(3), where preferential partitioning into the gel-phase has been reported. ${ }^{42,43}$ However, it should be noted that in these studies, the dye was incorporated into biphasic vesicles and introduced already in the dried dye-lipid mix (i.e. before the lipid vesicles were formed). In our study, the dye was added in the aqueous solution surrounding pre-made LUVs in order to reflect the experimental procedure that must be adopted when labeling cell-derived vesicles and other biological particles. Thus, while it might very well be that SP-DiO partitioning into the gel phase is thermodynamically most favored, especially considering that both DSPC and SP-DiO have saturated acyl chains, it is possible that dye incorporation efficiency (measured in our case) is rather dictated by insertion kinetics. Furthermore, it cannot be excluded that the dye's head group, in particular its charge or bulkiness (which are different from those of DiIC18(3)) influence the preference for a liquid disordered or a liquid ordered phase, which has also been reported by others. ${ }^{44,45}$

Additionally, we observed that the presence of lipid unsaturations leads to the emergence of a red-edge shoulder around $545 \mathrm{~nm}$ (Fig. 3c and ESI, $\dagger$ Fig. S2). Note that this shoulder is present in all emission spectra, but less apparent in LUVs with saturated lipids. It is possible that a ratiometric measure of the (a)

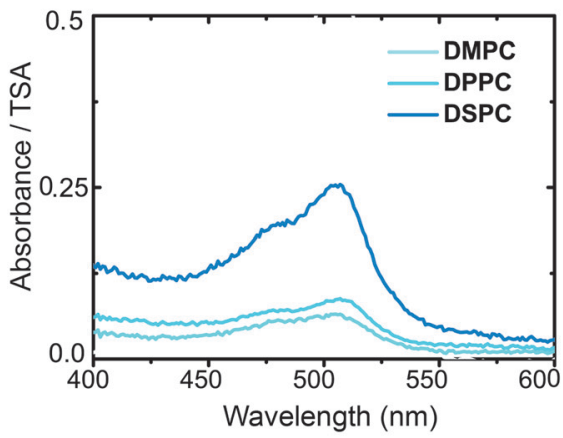

(b)

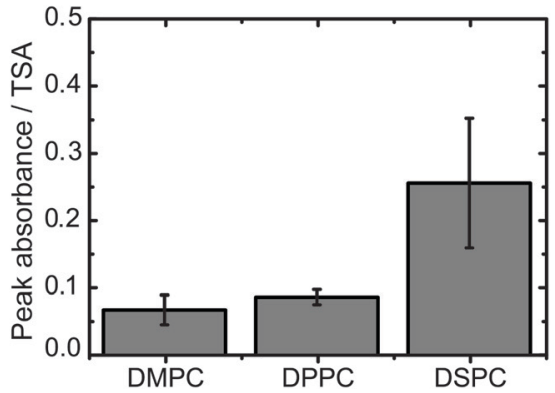

(c)



(d)

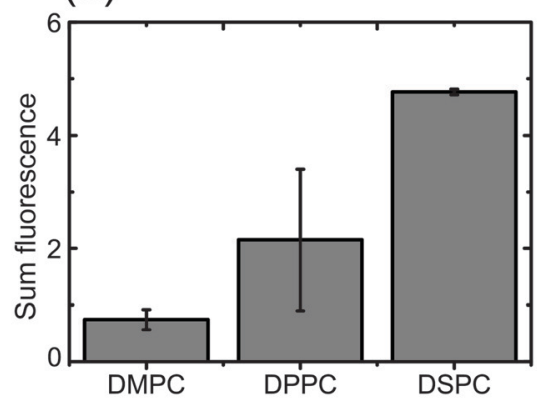

(e)

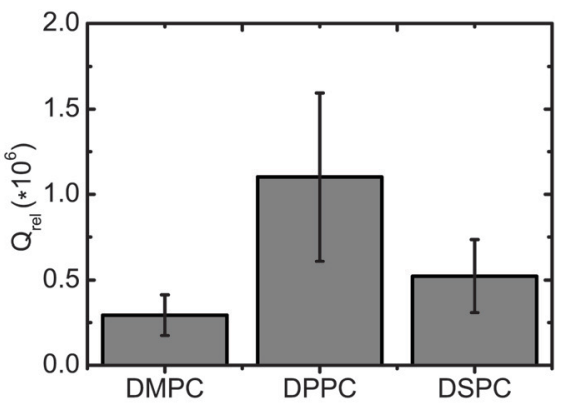

(f)

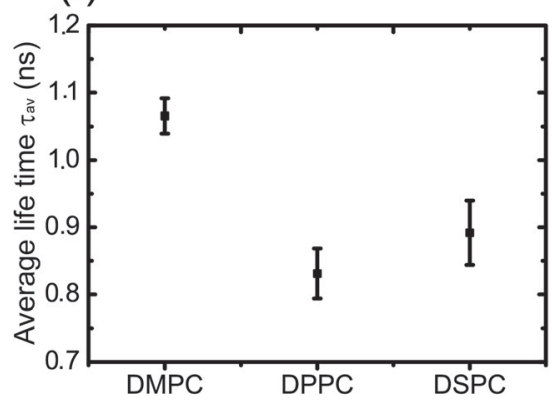





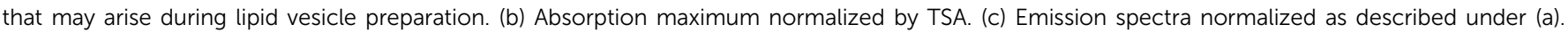

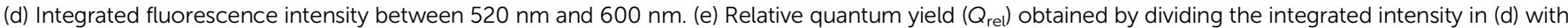


TCSPC decay curves. Error bars represent standard error of mean; experiments were repeated three times $(n=3)$ on different LUV batches. 
(a)

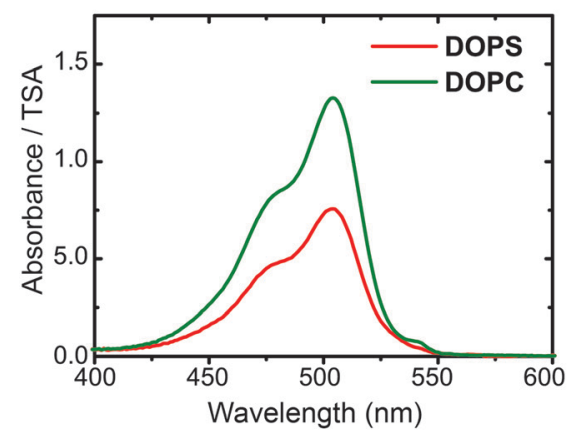

(b)

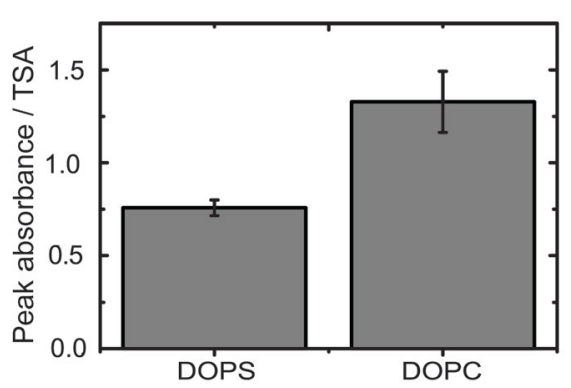

(c)

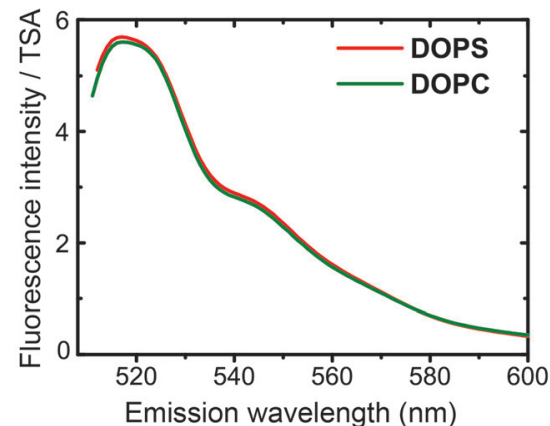

(d)

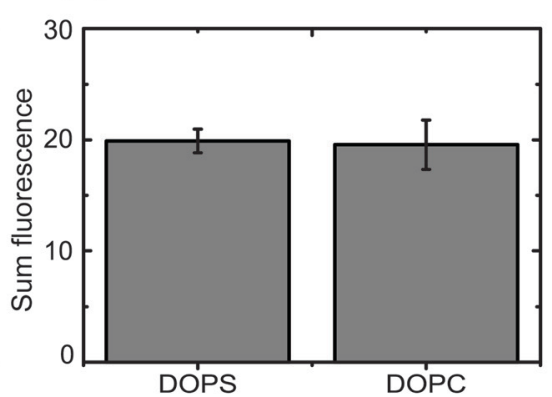

(e)

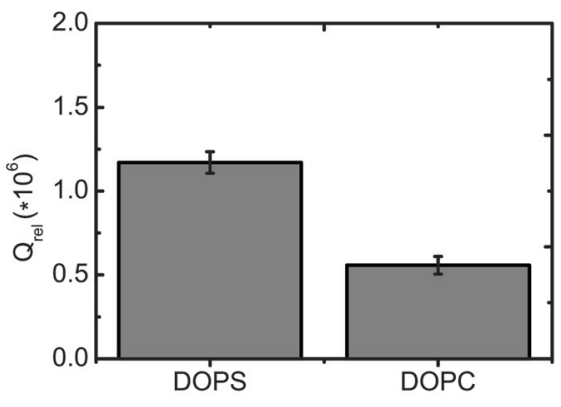

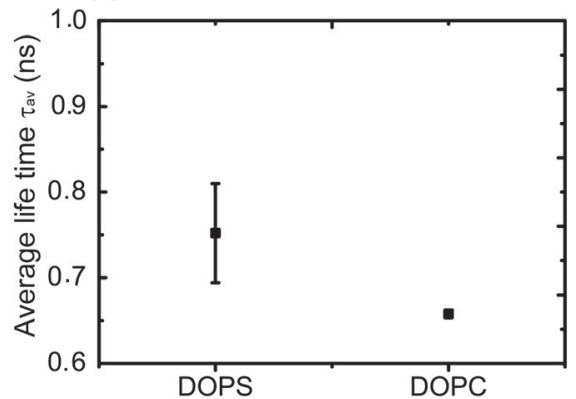

Fig. 5 Influence of lipid head-group charge. Absorption spectra of SP-DiO in LUVs of different compositions. The absorbance has been normalized by the total surface area (TSA) of the LUVs as measured by NTA (see ESI, $\uparrow$ Fig. S4b fir the individual values) to account for differences in lipid concentration that may arise during lipid vesicle preparation. (b) Absorption maximum normalized by TSA. (c) Emission spectra normalized as described under (a). (d) Integrated fluorescence intensity between $514 \mathrm{~nm}$ and $600 \mathrm{~nm}$. (e) Relative quantum yield $\left(Q_{\text {rel }}\right)$ obtained by dividing the integrated intensity in (d) with the dye concentration obtained from the peak absorbance shown in (b). (f) Average excited state lifetime determined from a double exponential fitting of the TCSPC decay curves. Error bars represent standard error of mean; experiments were repeated three times $(n=3)$ on different LUV batches.

intensities in the two peaks could be utilized to determine saturation in a lipid membrane of unknown composition.

\subsection{Acyl chain length}

Next, we investigated the influence of the phospholipid acyl chain length on SP-DiO binding and fluorescence, comparing LUVs made of the saturated phospholipids DMPC, DPPC and DSPC, which have 14, 16 and 18 carbon atoms per acyl chain respectively (Fig. 2 and Table 1). From absorption measurements (Fig. 4a and 4b), it is clear that incorporation of SP-DIO into this series of LUVs is dependent on the acyl chain length, although the correlation is not linear. Incorporation into DMPC and DPPC LUVs occurs at about equal efficiencies, whereas the absorption measurements indicate that considerably more dye has bound to the DSPC LUVs. This is consistent with the notion that introduction of dye molecules with mismatching alkylchains leads to perturbations in phospholipid chain packing and therefore in decrease of inter-chain interactions ${ }^{16,43,46}$ and suggests that the hydrophobic match of the 18 carbon tail in SP-DiO with the 18 carbon tail in DSPC is highly important here. The variation in total fluorescence intensity of SP-DiO in LUVs qualitatively correlates better with the variation in acyl chain length (Fig. 4c and 4d) than the absorption (Fig. 4a and $4 \mathrm{~b}$ ). Interestingly, SP-DiO inserted into DMPC lipid vesicles has the longest fluorescence lifetime of all the investigated systems, while at the same time having the lowest relative quantum yield (Fig. 4e and 4f).

\subsection{Lipid head group charge}

To investigate the role of lipid head group charge on the binding and fluorescence of SP-DiO, zwitterionic DOPC LUVs were compared to negatively charged DOPS LUVs (Fig. 2). Absorption measurements showed that SP-DiO incorporation into DOPS LUVs was reduced by approximately $50 \%$ compared to DOPC LUVs (Fig. $5 a$ and b). This is likely due to the electrostatic repulsion between the negative charges on the sulphonated dye and the negative head group charge of the DOPS lipids. However, SP-DiO exhibited similar total fluorescence intensity in both LUV types (Fig. 5d), resulting in a difference in relative quantum yield (Fig. 5e). Again, this is consistent with measured differences in the average fluorescence lifetime of SP-DiO, which is distinctively longer in DOPS LUVs (Fig. 5f). This effect may be ascribed to a local decrease in mobility of the fluorophore in the head group regions, as a result of the higher molecular packing of DOPS lipids compared to $\mathrm{DOPC}^{47}$ or due to deeper insertion of the dye into the bilayer due to charge repulsion, resulting in better shielding from water.

\subsection{Cholesterol}

Cholesterol is a major component of biological membranes which affects their structure and fluidity. In phosphocholine-rich 
(a)

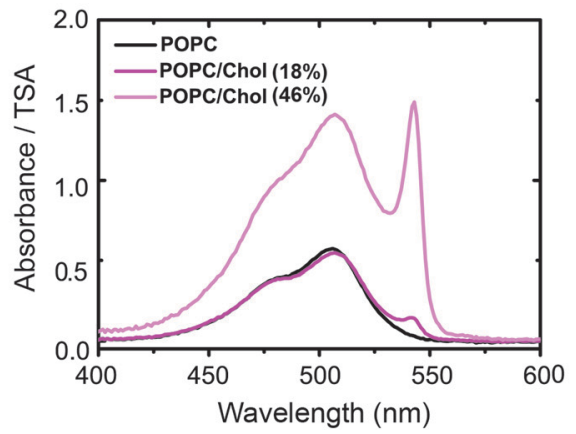

(b)

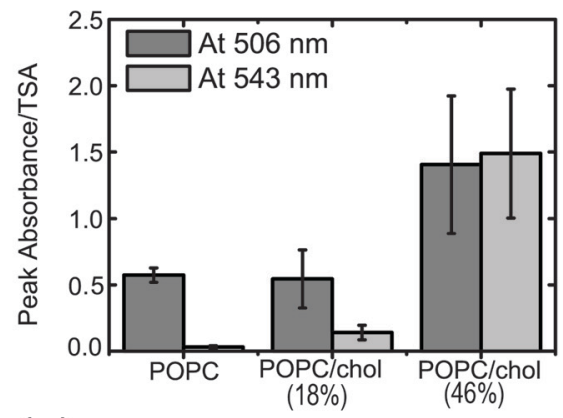

(g)

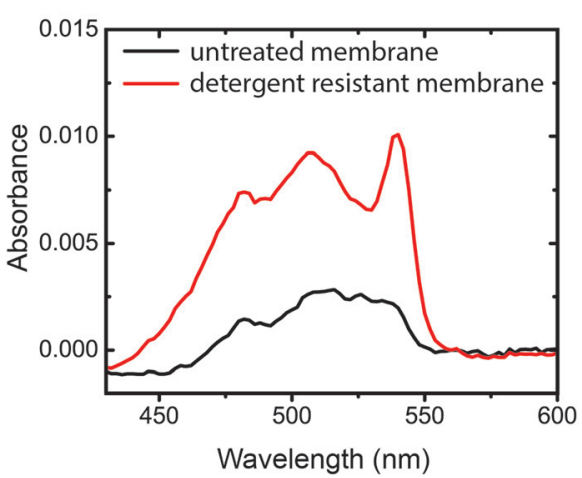

(c)

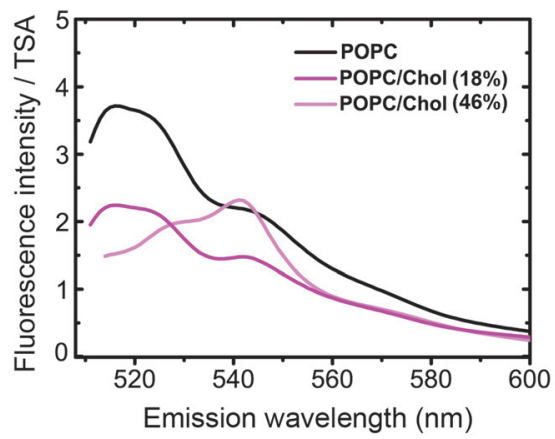

(d)

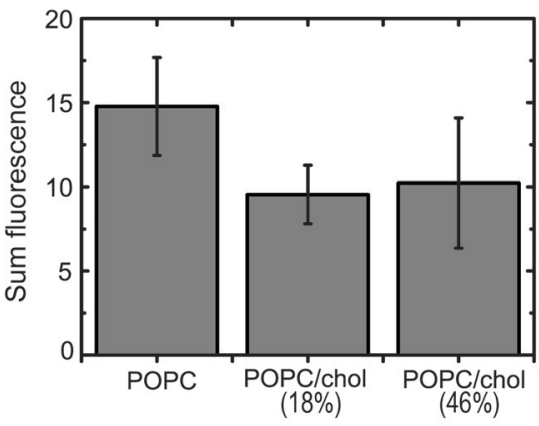

(e)

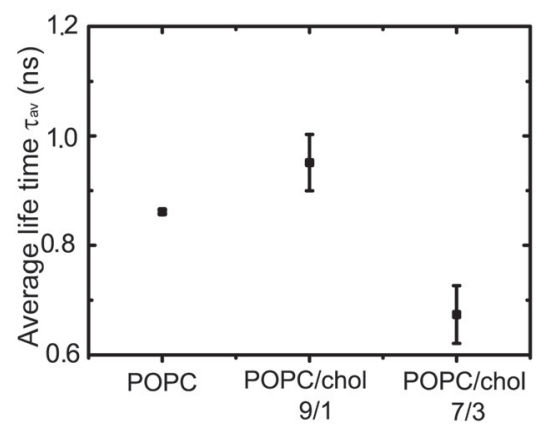

(f)

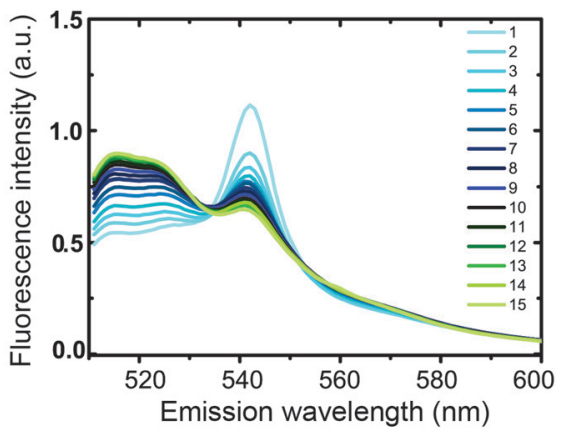

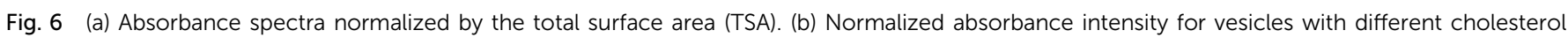

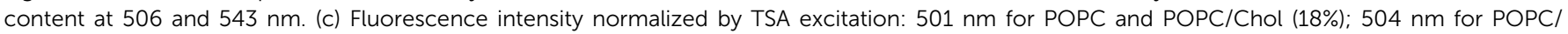

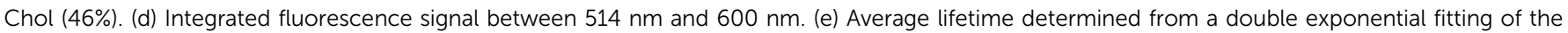

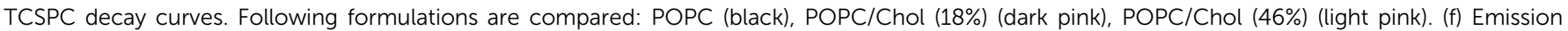

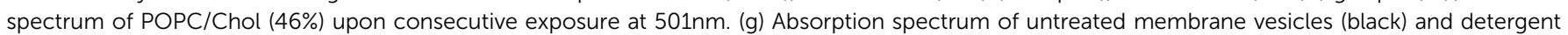

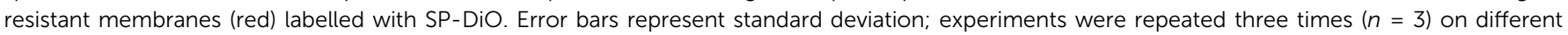
LUV batches.

membranes, it drives the formation of liquid ordered phases, characterized by a lower lipid diffusion coefficient $(\sim 1.5 \times$ lower for $50 \%$ cholesterol $)^{48}$ and increased membrane rigidity. ${ }^{49}$ To further assess the influence of lipid phase and cholesterol-rich domains on the binding and fluorescence of SP-DiO, we therefore investigated the effect of incorporation of cholesterol into POPC LUVs. In this case, POPC vesicles containing either 0,18 or $46 \mathrm{~mol} \%$ cholesterol were used. At room temperature, the membranes of these LUVs will be in either liquid disordered $\left(\mathrm{L}_{\mathrm{d}}\right)$ phase for pure POPC, exhibit a mixture of liquid disorder $\left(\mathrm{L}_{\mathrm{d}}\right)$ and liquid ordered $\left(\mathrm{L}_{\mathrm{o}}\right)$ phases for LUVs containing $18 \mathrm{~mol} \%$ cholesterol, or be mostly in the $\mathrm{L}_{\mathrm{o}}$ phase in LUVs containing 46 mol\% cholesterol. ${ }^{50}$ As shown in Fig. 6a, increasing the cholesterol content from $18 \%$ to $46 \mathrm{~mol} \%$ results in a significant increase in absorption $(2.5 \times)$, as well as in the appearance of a new red-shifted peak in the SP-DIO absorption spectrum (543 nm). (Fig. 6c) Such a spectral shift was specific to SP-DiO and did not appear in DiO C18(3), a similar membrane dye lacking the sulfophenyl groups (ESI, $\dagger$ Fig. S7). Similar, but less extensive red shifts have been observed with a nitrobenzooxadiazol (NBD) dye in LUVs maintained above and below the gel-to-liquid crystalline phase transition temperature and has 
been attributed to FRET from dye monomers to dye aggregates of J-type formed within the bilayer. ${ }^{51}$ This behavior is consistent with the appearance of the sharp absorption peak (Fig. 6a).

In addition to the appearance of a red-shift peak in the SP-DiO absorbance, the addition of cholesterol lead to a red-shift in the emission spectra of the dye where the peak at $543 \mathrm{~nm}$, becomes more pronounced when the cholesterol content is increased. (Fig. 6c). Interestingly, upon acquisition of several consecutive emission spectra, using an excitation wavelength of $501 \mathrm{~nm}$ for excitation, the $543 \mathrm{~nm}$ peak gradually disappears, concomitant with a regain in intensity in the $505 \mathrm{~nm}$ peak (Fig. 6f). Such an effect is not observed for the POPC and 18\% cholesterol LUVs which are mainly affected by small amounts of bleaching upon consecutive exposure of the sample (ESI, $\dagger$ Fig. S8). The existence of an isosbestic point in the exposure series indicate that this transformation occurs between two distinct states of the SP-DiO dye, one of which could well be a J-aggregate. The total SP-DiO fluorescence in LUVs with cholesterol is lower than in pure POPC vesicles (Fig. 6d); a finding that does not correlate with the relative levels of absorption (Fig. 6b). In the case of LUVs with $46 \mathrm{~mol} \%$ cholesterol, this can be explained by the shorter average fluorescence lifetime (Fig. 6e), but the origin of the effect for $18 \mathrm{~mol} \%$ cholesterol is less clear.

The SP-DiO spectral shifts observed in cholesterolcontaining LUVs may be relevant when labeling biological particles containing cholesterol-rich liquid ordered phases. In particular, the appearance of a new peak observed in the absorption spectrum is interesting as it opens new possibilities to use the SP-DiO dye as an indicator for the presence of cholesterol and liquid ordered phases, possibly even as a cholesterol concentration indicator in the analysis of biological membranes. This is further illustrated in Fig. $6 \mathrm{~g}$ which compares absorbance spectra of SP-DiO labelled native membrane vesicles produced from HeLa cells using either Triton X-100 treatment to produce detergent resistant membrane vesicles or without detergent treatment. Detergent resistant membrane fractions are well-known to have high cholesterol content ${ }^{52,53}$ relative to whole plasma membrane fractions, and accordingly the characteristic peak at $543 \mathrm{~nm}$ was observed to be larger for the detergent resistant membrane vesicles than whole untreated membrane vesicles.

\section{Conclusions}

We have performed a systematic investigation of the interplay between lipid environment and the insertion and fluorescence characteristics of the sulphonated carbocyanine dye SP-DiO, which is widely used to label synthetic as well as biological lipid membranes. Our data demonstrates clearly that lipid bilayer fluidity is an important determinant for effective dye insertion, but also that hydrophobic mismatch between the dye's acyl chain and the membrane lipids has strong adverse effects on intercalation. Furthermore, our results show that the chemical properties of lipids have a significant impact on the brightness of incorporated dye molecules and rationalize this effect with help of fluorescence lifetime variations. The study highlights the importance of carefully analyzing the dye's behavior when interpreting results from experiments involving extrinsic labeling of artificial as well as native biological membranes and points out complications with quantitative analysis of fluorescence intensity signals. We also demonstrate how the complex photophysics of SP-DiO could be an advantage, providing possibilities to probe membrane composition and alterations thereof, particularly in relation to cholesterol. Altogether, this study provides new information on the partitioning behavior and photophysics of a common carbocyanine dye that are of outermost importance to account for in analysis relying on quantitative fluorescence and when studying compositionally heterogeneous samples, including extracellular vesicles. Such systematic investigations are also of relevance in the context of the development of protocols to efficiently label biological vesicles extrinsically.

\section{Conflicts of interest}

There are no conflicts of interest to declare.

\section{Acknowledgements}

The Swedish Research Council (grant 2017-04029 to M. B., grant 2016-03902 to E. E.), the Wallenberg Foundation (M. B.), the Wenner-Gren foundations (E. E., Q. L.) and the Area of Advance Nanoscience and Nanotechnology and the Area of Advance Life Science Engineering and the Area of Advance Material Science (Chalmers) are acknowledged for financial support. The laboratory of Richard Lundmark (Umeå University) is acknowledged for help with the production of the native membrane material.

\section{References}

1 E. C. Jensen, Anat. Rec., 2012, 295, 2031-2036.

2 A. Kyrychenko, Methods Appl. Fluoresc., 2015, 3, 042003.

3 A. S. Klymchenko and R. Kreder, Chem. Biol., 2014, 21, 97-113.

4 L. A. Bagatolli and D. Needham, Chem. Phys. Lipids, 2014, 181, 99-120.

5 L. A. Bagatolli, Biomembranes, 2006, 1758, 1541-1556.

6 D. B. Vieira and L. F. Gamarra, Int. J. Nanomed., 2016, 11, 5381-5414.

7 P. Gangadaran, C. M. Hong and B. C. Ahn, Front. Pharmacol., 2018, 9, 169.

8 B. Brandenburg and X. W. Zhuang, Nat. Rev. Microbiol., 2007, 5, 197-208.

9 H. M. van der Schaar, M. J. Rust, C. Chen, H. van der EndeMetselaar, J. Wilschut, X. W. Zhuang and J. M. Smit, PLoS Pathog., 2008, 4, e1000244. 
10 L. W. Chu, Y. L. Huang, J. H. Lee, L. Y. Huang, W. J. Chen, Y. H. Lin, J. Y. Chen, R. Xiang, C. H. Lee and Y. H. Ping, J. Biomed. Opt., 2014, 19, 011018.

11 S. T. Chuo, J. C. Chien and C. P. Lai, J. Biomed. Sci., 2018, 25, 91.

12 T. Tian, Y. L. Zhu, F. H. Hu, Y. Y. Wang, N. P. Huang and Z. D. Xiao, J. Cell. Physiol., 2013, 228, 1487-1495.

13 C. A. Franzen, P. E. Simms, A. F. Van Huis, K. E. Foreman, P. C. Kuo and G. N. Gupta, BioMed Res. Int., 2014, 619829.

14 A. Morales-Kastresana, B. Telford, T. A. Musich, K. McKinnon, C. Clayborne, Z. Braig, A. Rosner, T. Demberg, D. C. Watson, T. S. Karpova, G. J. Freeman, R. H. DeKruyff, G. N. Pavlakis, M. Terabe, M. Robert-Guroff, J. A. Berzofsky and J. C. Jones, Sci. Rep., 2017, 7, 1878.

15 M. Deleers, J. P. Servais, F. Delaveleye and E. Wulfert, Biochem. Biophys. Res. Commun., 1984, 123, 178-185.

16 B. S. Packard and D. E. Wolf, Biochemistry, 1985, 24, 5176-5181.

17 L. M. B. B. Estronca, M. J. Moreno, M. S. C. Abreu, E. Melo and W. L. C. Vaz, Biochem. Biophys. Res. Commun., 2002, 296, 596-603.

18 A. Pokorny, P. F. Almeida and W. L. Vaz, Biophys. J., 2001, 80, 1384-1394.

19 J. E. Shaw, R. F. Epand, R. M. Epand, Z. G. Li, R. Bittman and C. M. Yip, Biophys. J., 2006, 90, 2170-2178.

20 W. Y. Leung, F. Mao, R. P. Haugland and D. H. Klaubert, Bioorg. Med. Chem. Lett., 1996, 6, 1479-1482.

21 M. G. Honig and R. I. Hume, J. Cell Biol., 1986, 103, 171-187.

22 B. Ragnarson, L. Bengtsson and A. Haegerstrand, Histochemistry, 1992, 97, 329-333.

23 A. Ferrari, D. Hannouche, K. Oudina, M. Bourguignon, A. Meunier, L. Sedel and H. Petite, J. Biomed. Mater. Res., 2001, 56, 361-367.

24 T. Wang and H. R. Petty, Cytometry, 1993, 14, 16-22.

25 E. J. van der Vlist, E. N. Nolte't Hoen, W. Stoorvogel, G. J. Arkesteijn and M. H. Wauben, Nat. Protoc., 2012, 7, 1311-1326.

26 S. S. Kanwar, C. J. Dunlay, D. M. Simeone and S. Nagrath, Lab Chip, 2014, 14, 1891-1900.

27 S. Joshi, R. P. Singh-Moon, J. A. Ellis, D. B. Chaudhuri, M. Wang, R. Reif, J. N. Bruce, I. J. Bigio and R. M. Straubinger, Neurosurgery, 2015, 76, 92-100.

28 A. Konno, N. Matsumoto and S. Okazaki, Sci. Rep., 2017, 7, 10089.

29 A. A. Janeczek, E. Scarpa, M. H. Horrocks, R. S. Tare, C. A. Rowland, D. Jenner, T. A. Newman, R. O. Oreffo, S. F. Lee and N. D. Evans, Nanomedicine, 2017, 12, 845-863.
30 Y. Yang, Y. Hong, G. H. Nam, J. H. Chung, E. Koh and I. S. Kim, Adv. Mater., 2017, 29, 1605604.

31 K. Kristensen, N. Ehrlich, J. R. Henriksen and T. L. Andresen, Langmuir, 2015, 31, 2472-2483.

32 P. J. Sims, A. S. Waggoner, C. H. Wang and J. F. Hoffman, Biochemistry, 1974, 13, 3315-3330.

33 D. Axelrod, Biophys. J., 1979, 26, 557-573.

34 R. R. Gullapalli, M. C. Demirel and P. J. Butler, Phys. Chem. Chem. Phys., 2008, 10, 3548-3560.

35 S. Jõemetsa, P. Joyce, Q. Lubart, M. Mapar, E. Celauro, B. Agnarsson, S. Block, M. Bally, E. Esbjörner, G. Jeffries and F. Höök, 2020, submitted.

36 N. Kucerka, M. P. Nieh and J. Katsaras, Biochim. Biophys. Acta, 2011, 1808, 2761-2771.

37 G. Lindblom and G. Oradd, Biomembranes, 2009, 1788, 234-244.

38 F. Harb, A. Simon and B. Tinland, Eur. Phys. J. E: Soft Matter Biol. Phys., 2013, 36, 140.

39 T. Tabouillot, H. S. Muddana and P. J. Butler, Cell. Mol. Bioeng., 2011, 4, 169-181.

40 A. Suhaj, A. Le Marois, D. J. Williamson, K. Suhling, C. D. Lorenz and D. M. Owen, Phys. Chem. Chem. Phys, 2018, 20, 16060-16066.

41 M. W. Baig, M. Pederzoli, P. Jurkiewicz, L. Cwiklik and J. Pittner, Molecules, 2018, 23, 1707.

42 R. D. Klausner and D. E. Wolf, Biochemistry, 1980, 19, 6199-6203.

43 C. H. Spink, M. D. Yeager and G. W. Feigenson, Biochim. Biophys. Acta, 1990, 1023, 25-33.

44 P. Sengupta, A. Hammond, D. Holowka and B. Baird, Biomembranes, 2008, 1778, 20-32.

45 E. Sezgin, I. Levental, M. Grzybek, G. Schwarzmann, V. Mueller, A. Honigmann, V. N. Belov, C. Eggeling, U. Coskun, K. Simons and P. Schwille, Biomembranes, 2012, 1818, 1777-1784.

46 T. Baumgart, G. Hunt, E. R. Farkas, W. W. Webb and G. W. Feigenson, Biomembranes, 2007, 1768, 2182-2194.

47 T. N. Murugova and P. Balgavy, Phys. Chem. Chem. Phys., 2014, 16, 18211-18216.

48 A. Filippov, G. Oradd and G. Lindblom, Biophys. J., 2003, 84, 3079-3086.

49 F. R. Svensson, P. Lincoln, B. Norden and E. K. Esbjorner, J. Phys. Chem. B, 2007, 111, 10839-10848.

50 R. F. M. de Almeida, A. Fedorov and M. Prieto, Biophys. J., 2003, 85, 2406-2416.

51 H. Sasaki and S. H. White, Biophys. J., 2009, 96, 4631-4641. 52 A. I. Magee and I. Parmryd, Genome Biol., 2003, 4, 234.

53 L. J. Pike, X. Han, K. N. Chung and R. W. Gross, Biochemistry, 2002, 41, 2075-2088. 